\title{
Crystal Structure of DsbA from Corynebacterium diphtheriae and Its Functional Implications for CueP in Gram-Positive Bacteria
}

\author{
Si-Hyeon Um ${ }^{1,3}$, Jin-Sik Kim ${ }^{1,3}$, Saemee Song ${ }^{1}$, Nam Ah Kim², Seong Hoon Jeong', and Nam-Chul Ha, *
}

\begin{abstract}
In Gram-negative bacteria in the periplasmic space, the dimeric thioredoxin-fold protein DsbC isomerizes and reduces incorrect disulfide bonds of unfolded proteins, while the monomeric thioredoxin-fold protein DsbA introduces disulfide bonds in folding proteins. In the Gram-negative bacteria Salmonella enterica serovar Typhimurium, the reduced form of CueP scavenges the production of hydroxyl radicals in the copper-mediated Fenton reaction, and DsbC is responsible for keeping CueP in the reduced, active form. Some DsbA proteins fulfill the functions of DsbCs, which are not present in Gram-positive bacteria. In this study, we identified a DsbA homologous protein (CdDsbA) in the Corynebacterium diphtheriae genome and determined its crystal structure in the reduced condition at $1.5 \AA$ resolution. CdDsbA consists of a monomeric thioredoxin-like fold with an inserted helical domain and unique $\mathrm{N}$-terminal extended reaion. We confirmed that CdDsbA has disulfide bond isomerase/reductase activitv, and we present evidence that the $\mathrm{N}$-terminal extended reqion is not required for this activitv and foldina of the core DsbA-like domain. Furthermore, we found that $C d D s b A$ could reduce CueP from C. diphtheriae.
\end{abstract}

\section{INTRODUCTION}

In Gram-negative bacteria, the Dsb (disulfide bond) family of proteins with the CXXC motif in the thioredoxin (TRX)-fold domain is involved in the formation and/or rearrangement of disulfide bonds of substrate proteins located in the periplasm (Cho and Collet, 2013; Martin, 1995). In Escherichia coli, there are two distinct pathways of the Dsb family of proteins in the periplasm, namely, the DsbA-DsbB oxidative pathway and

${ }^{1}$ Department of Agricultural Biotechnology, Center for Food Safety and Toxicology, Center for Food and Bioconvergence, Research Institute for Agricultural and Life Sciences, Seoul National University, Seoul 151-921, Korea, ${ }^{2}$ College of Pharmacy, Dongguk University-Seoul, Goyang 410820 , Korea, ${ }^{3}$ These authors contributed equally to this work.

*Correspondence: hanc210@snu.ac.kr

Received 9 April, 2015; revised 7 May, 2015; accepted 11 May, 2015; published online 17 June, 2015

Keywords: CueP, disulfide, DsbA, gram-positive bacteria
DsbC-DsbD isomerization/reductive pathway (Cho and Collet, 2013; Nakamoto and Bardwell, 2004). DsbA introduces disulfide bonds into folding proteins via the donation of the energetically unstable disulfide bond of DsbA, which in turn is reoxidized by the action of the membrane protein DsbB, a ubiquinone reductase (Hatahet et al., 2014). The crystal structure of DsbA from Escherichia coli $(E c D s b A)$ revealed a monomeric TRX-like fold domain with an inserted helical domain (Cho and Collet, 2013; Martin et al., 1993). EcDsbA consists of an unstable disulfide at the active site $C X X C$ motif which is characterized by a low pKa of the first nucleophilic cysteine residue in the CXXC motif and a high redox potential (Huber-Wunderlich and Glockshuber, 1998). In contrast, the V-shaped dimeric protein $\mathrm{DsbC}$ isomerizes or reduces incorrect disulfides in misfolded substrate proteins through the action of the reactive thiol motif (usually CXYC motif), which is kept in the reduced state by the membrane protein DsbD (Goldstone et al., 2001). DsbA has been considered a virulence factor as it catalyzes the folding of a number of bacterial virulence proteins (Heras et al., 2009).

Gram-positive bacteria do not have a conventional periplasm, but they do have a counterpart compartment confined between the plasma membrane and the cell wall (Matias and Beveridge, 2006). DsbA homologue proteins have been identified and characterized in some Gram-positive bacteria, and appear to be anchored to the outer leaflet of the plasma membrane (Kouwen et al., 2007). On the other hand, no DsbC homologues have been identified in Gram-positive bacteria. Staphylococcus aureus, a human pathogen, encodes only a DsbA-like protein $(\mathrm{SaDsbA})$ and lacks other Dsb proteins (Dumoulin et al., 2005), whereas Bacillus substilis have DsbB-like proteins $(\mathrm{BdbC})$ as well as a DsbA-like protein $(\mathrm{BdbD})$ (Dorenbos et al., 2002; Erlendsson and Hederstedt, 2002; Meima et al., 2002; Sarvas et al., 2004; Stein, 2005). The crystal structures of $\mathrm{SaDsbA}$ and $\mathrm{BdbD}$ are similar to that of $E c \mathrm{DsbA}$, despite low sequence identities (17\%,11\%, respectively) (Crow et al., 2009; Heras et al., 2008). However a tyrosine at the CXXC motif (resulting in CXYC) and Thr preceding the cis-Pro motif present in $\mathrm{SaDsbA}$, are more commonly found in DsbC homologues. Similar to $E c D s b A, B d b D$ has an unstable disulfide bond in the $C X X C$ motif, which is reformed through the action of $\mathrm{BdbC}$, Conversely, the catalytic disulfide bond in SaDsbA is relatively stable (Crow et al., 2009; Heras et al., 2008).

The host defense system produces hydrogen peroxide and superoxide anion via NADPH oxidase in the phagosomes of macrophages (Babior, 1999; Haraga et al., 2008). Recent stud- 
ies have highlighted the importance of copper ions in the killing of invading pathogens in the phagosomes of activated macrophages and neutrophils (White et al., 2009). Macrophage phagosomes accumulate copper ion during bacterial infections, and these copper ions mediate the conversion of hydrogen peroxide to hydroxyl radicals via the Fenton reaction (Achard et al., 2012). However, some intracellular bacteria can counteract this copper-mediated immune response by up-regulating copper export and detoxifying genes. Furthermore, these activities have been suggested to be important determinants of pathogen virulence (Hodgkinson and Petris, 2012; Wolschendorf et al., 2011). The periplasmic copper binding protein CueP was initially found in Salmonella enterica serovar Typhimurium, and has been implicated in bacterial pathogenesis because of its ability to scavenge ROS both directly and through periplasmic $\mathrm{Cu}, \mathrm{Zn}$ superoxide dismutase (Osman et al., 2013; Yoon et al., 2013; 2014b). The crystal structure and subsequent biochemical studies suggested that the cysteine-rich cluster, consisting of three conserved cysteine residues, plays an important role in the function of CueP, and needs to be kept reduced in order to function properly (Yoon et al., 2013; 2014b). It was further reported that DsbC maintains CueP in an active form by reducing the disulfide bonds of conserved cysteine residues (Yoon et al., 2014a).

Corynebacterium diphtheriae is a Gram-positive bacterium that evolved to survive in macrophage phagosomes and can cause diphtheria. In this study, we identified genes encoding a DsbA homologous protein (CdDsbA) and a CueP homologous protein $(C d C u e P)$ in the $C$. diphtheriae genome and determined the crystal structure of $C d D s b A$ at the reduced state as well as the functional relationship between the two proteins.

\section{MATERIALS AND METHODS}

\section{Cloning, expression, and protein production}

A DNA fragment encoding a truncated forms (residues 32-289) of $\mathrm{CaDsbA}$ (lacking the predicted $\mathrm{N}$-terminal transmembrane helix) was amplified by PCR using the genomic DNA of $C$. diphtheriae as a template (Supplementary Table S1). The PCR products were ligated into cut pProEX-HTA vector (Invitrogen, USA) and transformed into E. coli XL1-blue cell. DNA cloning, protein expression, and protein purification of $S$. enterica serovar Typhimurium CueP (residues 22-179 based on the numbering of the precursor (CueP) have been previously described (Yun et al., 2011). Briefly, CoDsbA containing a Nterminal hexahistidine tag was expressed in E. coli BL21 (DE3) cells cultured in $1 \mathrm{~L} \mathrm{LB}$ media at $37^{\circ} \mathrm{C}$ and induced at $\mathrm{OD}_{600}=$ 0.7 by adding IPTG $(0.5 \mathrm{mM}$ final concentration). After incubation for $5 \mathrm{~h}$ at $30^{\circ} \mathrm{C}$, cells were harvested and resuspended in lysis buffer containing $20 \mathrm{mM}$ Tris (pH 8.0), $150 \mathrm{mM} \mathrm{NaCl}$, and $2 \mathrm{mM}$ 2-mercaptoethanol. The resuspended lysate was disrupted by sonication, and cell debris was removed by centrifugation. The supernatant was mixed with Ni-NTA affinity resin (Qiagen, USA) pre-incubated with lysis buffer, and the resulting mixture was stirred for $1.5 \mathrm{~h}$ at $4^{\circ} \mathrm{C}$. After the slurry was loaded onto the column, unbound proteins were washed off with lysis buffer supplemented with $20 \mathrm{mM}$ imidazole. Recombinant proteins were eluted with $30 \mathrm{ml}$ of lysis buffer supplemented with $250 \mathrm{mM}$ imidazole. Fractions containing CoDsbA were pooled, and the hexahistidine tag was cleaved with recombinant TEV protease by incubation at room temperature overnight. CoDsbA protein was purified using HiLoad Superdex 200 columns (GE Healthcare, USA) pre-equilibrated with lysis buffer. The purified protein was concentrated to $15 \mathrm{mg} / \mathrm{ml}$ in $20 \mathrm{mM}$ Tris buffer $(\mathrm{pH}$
8.0) containing $150 \mathrm{mM} \mathrm{NaCl}$ and $2 \mathrm{mM}$ 2-mercaptoethanol and then crystalized. For CaDsbA ${ }^{\triangle 32-95}$ (residues 96-289), a DNA fragment encoding the corresponding region was amplified by PCR using the resulting plasmid for CoDsbA (residues 32-289) as a template (Supplementary Table S1). The same procedures as used for the CaDsbA (residues 32-289) protein were applied to express and purify the protein.

To obtain selenomethionine-labeled CdDsbA, E. coli strain B834 (DE3) cells expressing the recombinant plasmid were cultured overnight in adapted $\mathrm{M} 9$ medium, which is containing $47 \mathrm{mM} \mathrm{Na}_{2} \mathrm{HPO}_{4}, 22 \mathrm{mM} \mathrm{KH}_{2} \mathrm{PO}_{4}, 18.7 \mathrm{mM} \mathrm{NH}_{4} \mathrm{Cl}, 8 \mathrm{mM} \mathrm{NaCl}$ and $30 \mathrm{mg} / \mathrm{ml} \mathrm{L}$-selenomethinone ( $\mathrm{TCl}$, Japan). The cells were collected by centrifugation (Supra 22K; Hanil Centrifuges, Korea). The pellet was resuspended in a lysis buffer containing $20 \mathrm{mM}$ Tris (pH 8.0), $150 \mathrm{mM} \mathrm{NaCl}$, and $2 \mathrm{mM}$ 2-mercaptoethanol. Gel-filtration chromatography using HiLoad Superdex 200 columns (GE Healthcare, USA) was subsequently performed. The purity of the protein samples was examined by SDS-PAGE.

Crystallization, data collection. and structure determination of selenomethionine-labeled CdDsbA

Selenomethionine-derivatized crystals of $\mathrm{CdDsbA}$ were obtained in a precipitation solution containing $0.2 \mathrm{M}$ sodium acetate, $0.1 \mathrm{M}$ Tris- $\mathrm{HCl} \mathrm{pH} 8.5$, and $30 \%$ PEG $2 \mathrm{~K}$ by mixing $1 \mu \mathrm{l}$ of protein solution with $1 \mu$ of precipitant solution. X-ray diffraction data of the crystals were collected at the $5 \mathrm{C}$ beamline of the Pohang Accelerator Laboratory (PAL, Korea) using a QUANTUM 315 CCD detector (ADSC). The diffraction data sets were handled and scaled with the HKL2000 package (Otwinosky and Minor, 1997). The crystal belonged to the space group $P 2_{1} 2_{1} 2_{1}$ with cell measurements of $a=48.6 \AA, b=68.2 \AA$, and $c=69.0 \AA$. Initial phases were determined by the single-wavelength anomalous dispersion (SAD) method (Table 1). Three selenium sites were found using the program AUTOSOL in the PHENIX program suite (Adams et al., 2002), and we obtained the excellent electron density map using the AUTOSOL SAD phasing at $1.8 \AA$ A resolution.

Crystallization, data collection, and structure determination of CdDsbA

CoDsbA protein $(15 \mathrm{mg} / \mathrm{ml})$ was crystallized by the same method as selenomethionine-derivatized CaDsbA. The crystal belonged to the space group $P 2_{1} 2_{1} 2_{1}$ with cell dimensions of $a$ $=48.8 \AA, b=68.4 \AA, c=69.1 \AA$, which was similar to that of selenomethionine-derivatized $\mathrm{CdDsbA}$. The structure was solved using coordinates obtained from the selenomethionine-derivatized CaDsbA structure (Table 1).

\section{Disulfide isomerase activity assay}

S. enterica serovar Typhimurium DsbC (StDsbC) was purified described previously (Jiao et al., 2013). Bovine pancreatic RNase A (Sigma-Aldrich, USA) was reduced and denatured in $7 \mathrm{M}$ guanidium chloride, $40 \mathrm{mM}$ Hepes ( $\mathrm{pH}$ 7.5), $120 \mathrm{mM}$ DTT, and $0.2 \mathrm{mM}$ EDTA at $25^{\circ} \mathrm{C}$ for $24 \mathrm{~h}$, and buffer-exchanged into $0.1 \%$ acetic acid by dialysis. The thiol content of reduced DsbC and DsbA proteins and the denatured and reduced RNase $A$ (drRNase) was measured with DTNB as described (Jiao et al., 2013), and they were found to be more than $90 \%$ reduced. Proteins were frozen and stored at $-70^{\circ} \mathrm{C}$ until use. To prepared RNase A with scrambled disulfide bonds (scRNase), the drRNase was oxidized with copper $/ \mathrm{H}_{2} \mathrm{O}_{2}$; drRNase was diluted into $50 \mathrm{mM}$ sodium phosphate buffer ( $\mathrm{pH} \mathrm{6.0)} \mathrm{containing} 300$ $\mathrm{mM} \mathrm{NaCl}$ to a final concentration of $50 \mu \mathrm{M}$ in the presence of $50 \mu \mathrm{M} \mathrm{CuCl}_{2}$ and $2 \mathrm{mM} \mathrm{H}_{2} \mathrm{O}_{2}$, or the buffer alone and incubated 
Table 1. X-ray data collection and refinement statistics

\begin{tabular}{|c|c|c|}
\hline Dataset & Native & Se-Met \\
\hline Wavelength $(\AA ̊)$ & 0.99998 & 0.97911 \\
\hline Resolution limit $(\AA ̊)$ & $50.0-1.5(1.55-1.5)$ & $50-1.80(1.83-1.80)$ \\
\hline Space group & $P 2_{1} 2_{1} 2_{1}$ & $P 2_{1} 2_{1} 2_{1}$ \\
\hline Unit cell $(\AA ̊)$ & $a=48.6 \AA$ & $a=48.8 \AA, b=68.4 \AA, c=69.1 \AA$ \\
\hline \multicolumn{3}{|l|}{ Reflections $^{1}$} \\
\hline Unique & 36144 & 41100 \\
\hline Redundancy & $10.6(8.4)$ & $6.1(5.4)$ \\
\hline$R_{\mathrm{sym}}(\%)^{1}$ & $5.1(22.2)$ & $5.7(12.5)$ \\
\hline Completeness (\%) ${ }^{1}$ & $99.8(99.9)$ & $99.7(99.7)$ \\
\hline Average $\mathrm{I} / \sigma^{1}$ & $71.4(9.6)$ & $60.0(21.4)$ \\
\hline \multicolumn{3}{|l|}{ Refinement } \\
\hline Resolution range $(\AA)$ & $20-1.5(1.54-1.5)$ & $30-1.8(1.83-1.8)$ \\
\hline$R$-factor (\%) & $16.9(16.3)$ & $16.7(15.9)$ \\
\hline$R_{\text {free }}(\%)^{2}$ & $19.3(19.4)$ & $20.4(20.4)$ \\
\hline Average $B$ value $\left(\AA^{2}\right)$ & 19.0 & 19.0 \\
\hline Wilsom $B$ value $\left(\AA^{2}\right)$ & 17.0 & 19.1 \\
\hline Rmsd for bonds $(\AA)$ & 0.006 & 0.006 \\
\hline Rmsd for angles $\left(^{\circ}\right)$ & 1.056 & 1.056 \\
\hline \multicolumn{3}{|l|}{ Ramachandran plot } \\
\hline Most favored & 98.9 & 98.5 \\
\hline Additionally favored & 1.1 & 1.5 \\
\hline Coordinate error $(\AA)$ & 0.12 & 0.16 \\
\hline PDB code & 4PWO & 4PWP \\
\hline
\end{tabular}

${ }^{1}$ The numbers in parentheses are statistics for the highest resolution shell.

${ }^{2} R_{\text {free }}$ was calculated with $5 \%$ of the dataset.

at $25^{\circ} \mathrm{C}$ for 30 min. $\mathrm{CuCl}_{2}$ and $\mathrm{H}_{2} \mathrm{O}_{2}$ were removed using a desalting column (HiPrep 26/10 Dsealting; GE Healthcare) in $50 \mathrm{mM}$ sodium phosphate buffer $(\mathrm{pH} 7.5)$ containing $300 \mathrm{mM}$ $\mathrm{NaCl}$. To test the disulfide isomerase activities of DsbC, DsbA, or 2-mercaptorethanol, scRNase $(5 \mu \mathrm{M})$ was incubated with the reduced forms of DsbC $(20 \mu \mathrm{M})$, DsbA $(20 \mu \mathrm{M})$, or 2-mercaptorethanol $(20 \mu \mathrm{M})$ at the room temperature. We performed an RNase A activity assay based on CCMP as a substrate (Hiniker et al., 2005) from $200 \mu \mathrm{l}$ aliquots of the samples which were taken in given times after the incubation. The aliquot $(200 \mu \mathrm{l})$ was added to $4 \mathrm{mM} \mathrm{cCMP}$ (final concentration) in $400 \mu \mathrm{l}$ of 300 $\mathrm{mM} \mathrm{NaCl}, 50 \mathrm{mM}$ sodium phosphate $(\mathrm{pH} 7.5)$ to measure the RNase A activity. The hydrolysis of cCMP was followed at 296 $\mathrm{nm}$ for $30 \mathrm{~s}$. The protein concentrations were determined by the specific absorbance at $280 \mathrm{~nm}$.

\section{Reductase assay of CdDsbA using NTB-labeled CdCueP}

S. enterica serovar Typhimurium CueP (StCueP) was purified described previously (Yun et al., 2011). CdCueP was cloned, expressed, and purified using the similar procedures as used for StCueP. Briefly, a DNA fragment of CdCueP (residues 29211), amplified from a genomic DNA of $C$. diphtheriae, was ligated into the BamHI and Hindlll sites of pProEX-HTA vector (Invitrogen, USA) (Supplementary Table S1). To prepare the reduced forms of CueP proteins and CdDsbA proteins in a reducing agent-free buffer, purified protein samples were dialyzed against degassed $20 \mathrm{mM}$ Tris buffer ( $\mathrm{pH}$ 8.0) containing $150 \mathrm{mM} \mathrm{NaCl}$ twice for $2.5 \mathrm{~h}$, and then immediately concentrated using a Centricon device (GE Healthcare, USA). To pre- pare NTB-labeled proteins, CueP proteins $(20 \mu \mathrm{M}, 5 \mathrm{ml})$ were first incubated with a 100-fold excess (2 mM) of DTT for $1 \mathrm{~h}$ at room temperature. Excess DTT was then removed using a HiPrep 26/10 desalting column (GE Healthcare) pre-equilibrated with $20 \mathrm{mM}$ Tris $\mathrm{HCl}(\mathrm{pH} \mathrm{8.0)}$ and $150 \mathrm{mM} \mathrm{NaCl}$. Proteins were then incubated with $4 \mathrm{mM}$ of the Ellman's reagent (DTNB) for 2 $\mathrm{h}$ at room temperature to produce 2-nitro-5-thiobenzoic acid (NTB)-labeled CueP proteins. Unreacted DNTB and free thiobis(2-ntirobenzoic acid) or TNB were removed using HiPrep 26/10 desalting columns with $20 \mathrm{mM}$ Tris $-\mathrm{HCl}(\mathrm{pH} 8.0)$ and $150 \mathrm{mM}$ $\mathrm{NaCl}$. Upon reduction of the mixed disulfide by $\mathrm{CdDsbA}$ or DTT, yellowish TNB was released. In the reductase assay, the NTBlabeled CueP or CoDsbA proteins $(5.0 \mu \mathrm{M})$ were reacted with reduced CdDsbA protein or CdCueP, or DTT $(15 \mu \mathrm{M})$ at room temperature. Absorbance of liberated TNB was measured at $412 \mathrm{~nm}$.

\section{Differential scanning calorimetry (DSC)}

DSC measurements were obtained using a VP-DSC Microcalorimeter (Microcal, USA) with $0.51471 \mathrm{~cm}^{3}$ twin cells for the reference and sample solutions. Prior to DSC measurements, the sample and reference were degassed under vacuum while being stirred. Next, $20 \mathrm{mM}$ Tris- $\mathrm{HCl}(\mathrm{pH}$ 8.0) containing 150 $\mathrm{mM} \mathrm{NaCl}$ and $2 \mathrm{mM}$ 2-mercaptoethanol prepared for dialysis were used as references to obtain baselines. Measurements were repeated three times at a scan rate of $1^{\circ} \mathrm{C} / \mathrm{min}$ for each sample. The temperature ranged from 15 to $110^{\circ} \mathrm{C}$ and the final thermogram was processed by subtracting the baseline from the sample thermogram. Thermal compensation curves were 
evaluated using the Microcal LLC DSC plug-in the Origin 7.0 software package provided with the DSC instrument. Transition melting points were calculated using the same software package.

\section{RESULTS}

\section{Structural determination}

It was previously reported that periplasmic isomerase DsbC plays a key role in maintaining CueP protein in a reduced state in S. enterica (Yoon et al., 2014a). C. dipththeriae does not have a DsbC homologue in the genome like other Grampositive bacteria (Kouwen et al., 2007). We identified a gene that encodes a DsbA homologous protein $(C d D s b A)$ in the $C$. diphtheriae genome as a putative functional substitute for DsbC.

DsbA from C. diphtheriae encodes a protein of 289 amino acid residues with a putative $\mathrm{N}$-terminal signal peptide (residues 1-21) for export out of the cytoplasmic membrane, which was predicted by SignalP (Petersen et al., 2011). The transmembrane prediction program TMHMM suggested that residues 12-31 were located in the transmembrane region, while residues 32 to the C-terminus were located on the outer plasma membrane (Chen et al., 2003). Moreover, BdbD, a functional and structural homologue of DsbA, was shown to be localized outside of the cytoplasmic membrane by its $\mathrm{N}$-terminal transmembrane segment (Crow et al., 2009). When we expressed a truncated protein lacking the predicted $\mathrm{N}$-terminal signal peptide and transmembrane segment, the protein was produced in a soluble form. Taken together, CoDsbA is likely anchored to the cytoplasmic membrane via its $\mathrm{N}$-terminal transmembrane segment similar to $\mathrm{BdbD}$, although this remains to be validated experimentally.

We obtained crystals of the truncated form of CaDsbA (residues 32-289) in the reduced condition. The crystal structure was determined using the SAD method with selenomethioninelabeled crystals (Rice et al., 2000). A high-quality electron density map was produced, and the majority of the model could be traced. The crystals belonged to the $P 2_{1} 2_{1} 2_{1}$ space group with a single molecule in the asymmetric unit (Table 1). The final model was refined against the $1.5 \AA$ resolution native dataset of both crystals in reduced form, and contained all residues between 38-287. The structure was refined to a free $R$ value of $19.5 \%$ with good stereochemistry. Further details on the structure determination and refinement are given in Table 1.

\section{Overall structure}

CaDsbA exhibited a one-domain structure with extended loops at both termini (Fig. 1A). The core DsbA-like domain of CaDsbA (residues 115-277) could be divided into two regions: a classical TRX-fold region (residues 115-156 and residues 229-277; colored in green), and an $\alpha$-helical region (residues 157-228; colored in salmon). The first part (residues 115-156) of the classical TRX-fold region formed a $\beta \alpha \beta$ component and the second part (residues 229-277) formed $\alpha \beta \beta \alpha$ components. The first and second parts of the TRX-fold region were interrupted by an $\alpha$-helical region (residues 157-228) forming four $\alpha$-helices. The helix $\alpha 7$ served as an extension of the helix $\alpha 8$ in the second part of the TRX-fold region (Fig. 1A). The N-terminal extended region (residues 32-114) consisted mainly of loops with two short $\alpha$-helices ( $\alpha 1$ and $\alpha 2$ ) attached to the core DsbA-like domain via a few polar interactions between the backbone and side chains or backbone. The side chain of Arg172 from $\alpha 5$ in the core domain formed a polar interaction with the backbone carbonyl groups of Ala80 and Ile82 from the $\mathrm{N}$-terminal region.

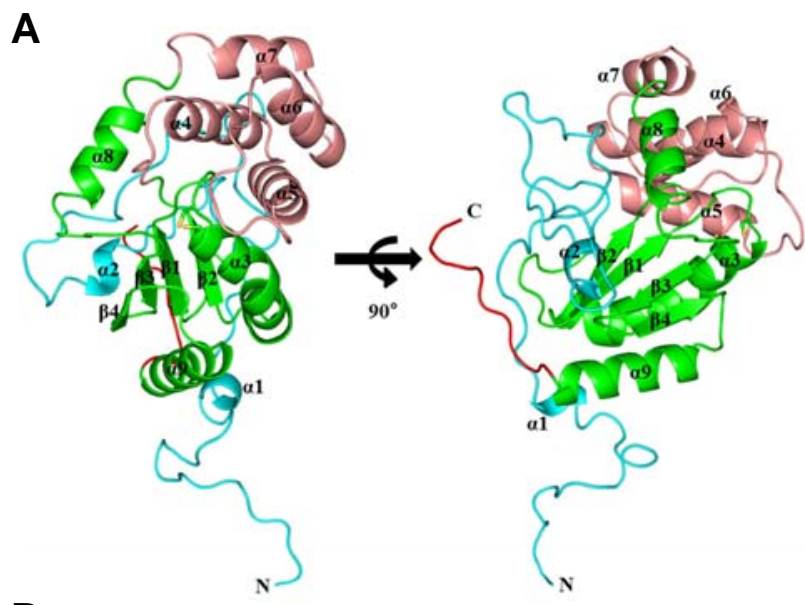

B

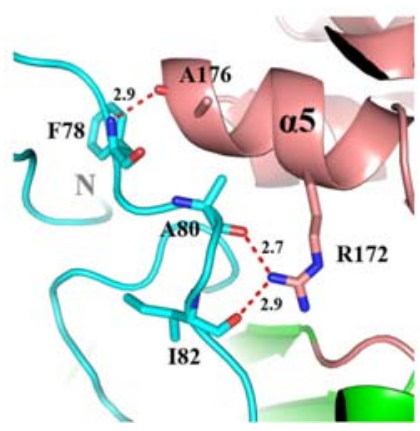

Fig. 1. Overall structure of $C d \mathrm{DsbA}$. (A) Orthogonal views of $\mathrm{CdDsbA}$ in the ribbon representations. CdDsbA consists of a core DsbA-like domain (green and salmon), an $\mathrm{N}$-terminal extended region (cyan), and a C-terminal tail region (red). The core DsbA-like domain is composed of a TRX-fold region (green) and intervening $\alpha$-helical region (salmon). (B) Interactions between the core DsbAlike domain and the $\mathrm{N}$-terminal extended region.

Likewise, the backbone $\mathrm{NH}$ group of Ala176 in the core domain formed a hydrogen bond with the backbone carbonyl group of Phe78 in the N-terminal region (Fig. 1B). In addition, the Cterminal stretched region (residues 278-289; Fig. $1 \mathrm{~A}$ colored in red) protruded from the core domain.

\section{Comparison with other DsbA family proteins}

We performed a sequence alignment with the DsbA family proteins, including the structurally and biochemically characterized EcDsbA, BdbD, and SaDsbA proteins (Crow et al., 2009; Heras et al., 2008; Martin et al., 1993) (Fig. 2A). We found that the $\mathrm{N}$-terminal extended region (residues 32-114; colored in cyan) and the C-terminal stretched region (residues 278-289) were unique to $C a D s b A$. In particular, the $\mathrm{N}$-terminal extended region was unusually long and its interactions with the core DsbA-like domain were not extensive. The homologous and core domain (residues 115-277; Fig. 2A) of the CaDsbA structure was superposed onto other DsbA family proteins, yielding rmsd values for BdbD, SaDsbA, and EcDsbA of 1.2, 2.3, and $4.8 \AA$, respectively. The structural superposition revealed a notable conformational difference in the loop connecting $\alpha$ helices $\alpha 5$ and $\alpha 6$ (residues 190-202; Figs. 2A and 2B; blue box). Specifically, the conformations of this connecting loop 
$\boldsymbol{A}$

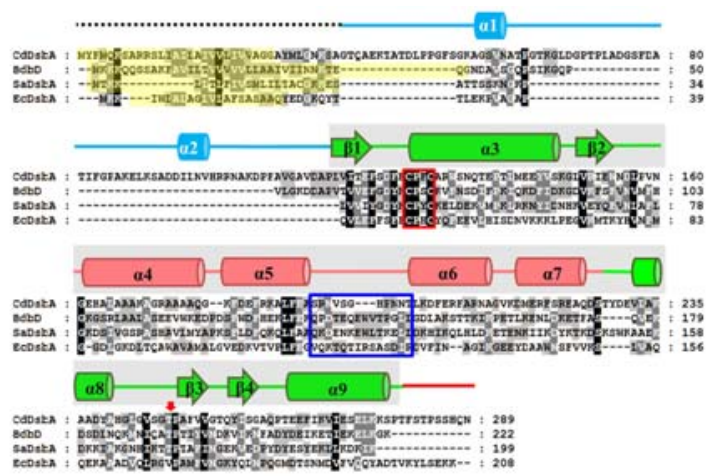

B

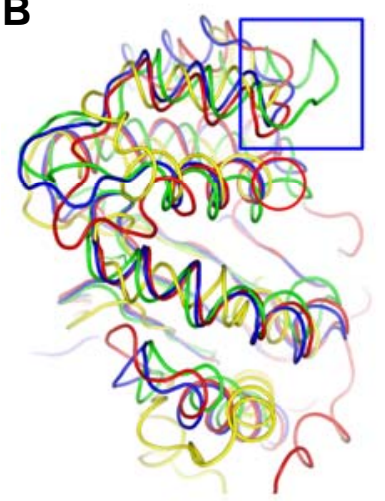

C
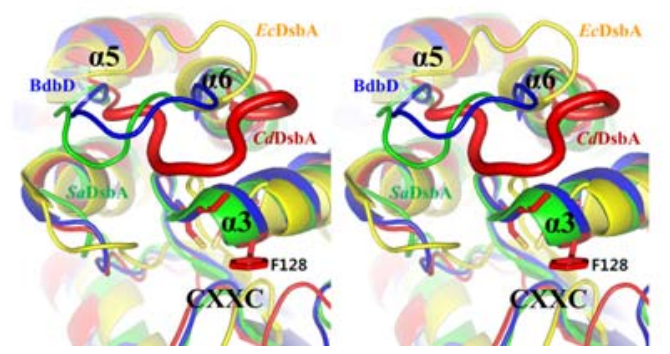

Fig. 2. Sequence and structural comparison. (A) Amino acid sequence alignment of $C d D s b A$ with BdbD (PDB code: $3 E U 3$ ), SaDsbA (PDB code: 3BCl), and EcDsbA (PDB code: 1A2L). Secondary-structure elements are depicted on the top line with the same color scheme as in Fig. 1. The yellow color indicated the signal peptide. The core DsbA-like domain is shaded in grey. The red box indicates the $C X X C$ motif, and the blue box indicates the loop connecting $\alpha 5$ and $\alpha 6$. The residues preceding the cis-Pro motif are indicated by a red arrow. (B) Structural superimposition of the core DsbA-like domains of CaDsbA (red), BdbD (blue), SaDsbA (green), and $E c D s b A$ (yellow), displayed in the stereo view. The blue box indicates the loop connecting $\alpha 5$ and $\alpha 6$. The $C X X C$ motif is indicated by a red circle. (C) Structural superimposition of the loop region of CaDsbA (red), BdbD (blue), SaDsbA (green), and EcDsbA (yellow), displayed in the stereo view.

were divergent among DsbA family proteins (Figs. 2B and $2 \mathrm{C}$ ). The connecting loop of $\mathrm{CdDsbA}$ was located in closest proximity to the CXXC motif (Fig. 2C).

In the case of $\mathrm{BdbD}$, a calcium binding site was identified that appeared to be involved in modulation of the oxidative properties of the active site cysteine residues in a calcium dependent manner (Crow et al., 2009). However, no such calcium binding

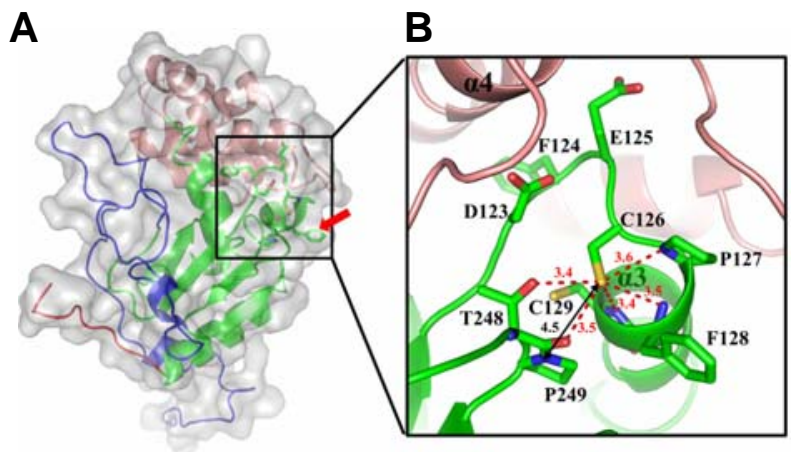

Fig. 3. Structural features of the CaDsbA active site. (A) Semitransparent surface representation overlaid on the CaDsbA structure. Phe128 is indicated by a red arrow. The boxed region is enlarged in (B). (B) The active site $C X X C$ motif of $C a D s b A$ is located in the third $\alpha$-helix ( $\alpha 3)$. The CXXC motif (Cys126-Pro127-Phe128Cys129) is located close to the Thr-cis-Pro (Thr258-Pro249) motif. The hydrogen bonds involving Cys $126 \mathrm{~S} \gamma$ are indicated by the broken lines. The distance between Cys126 S $\gamma$ and Pro249 is indicated.

site was found in CoDsbA.

\section{Structural features of the CdDsbA active site}

The cysteine residues in the active site $C X X C$ motif were present in the reduced form, as expected (Fig. 3A). The CXXC motif was located at the third $\alpha$-helix $(\alpha 3)$, and was found to be long and highly curved like other DsbA proteins (Shouldice et al., 2011). This helical curve seems to be caused by a proline residue (Pro138) in the middle of the helix. The sequence of the CXXC motif in CdDsbA was ${ }^{126} \mathrm{CPFC}^{129}$ (Fig. 2A). Interestingly, the second residue Pro127 in the $C X X C$ motif was conserved in $\mathrm{BdbD}, \mathrm{SaDsbA}$, and EcDsbA (CPSC in BdbD, CPYC in $S a D s b A$, and $C P H C$ in $E c D s b A$ ). It was unusual that the hydrophobic residue Phe128 in the CaDsbA CXXC motif was exposed to solvent unlike other DsbA proteins (Fig. 3B). The phenylalanine residue does not block the active site cysteine residues in the crystal structure. We speculate that Phe128 might play a role in recognition of the hydrophobic region on the surface of the substrates.

Like other TRX superfamily proteins, a conserved cis-Pro loop containing Pro249 was found in close proximity to the nucleophilic Cys residue (Cys126) (Fig. 3). It has been suggested that the residue preceding the cis-Pro loop is related to substrate specificity (Huber-Wunderlich and Glockshuber, 1998), and also that the residue preceding the cis-Pro is specifically involved in modulating the redox properties of TRX-like proteins. CaDsbA, SaDsbA, and BdbD all contain the Thr-cisPro motif, whereas EcDsbA has a Val-cis-Pro motif (Ren et al., 2009). Taken together, our findings suggest that $C d D s b A$ is more functionally related to $S a D s b A$ and $B d b D$ than $E c D s b A$.

It was reported that the thiolate forms at the nucleophilic cysteine residues (Cys30 in the $E c D s b A$ numbering) are stabilized in reduced forms of $E c \mathrm{DsbA}$ and Vibrio cholera DsbA (Guddat et al., 1998; Mohanty et al., 2012). The stabilization of the thiolate forms were suggested to result from the interactions within the CXXC moitf (Guddat et al., 1998). Thiol group and the backbone amide group of the resolving cysteine residue (Cys32 in the EcDsbA numbering), and the imidazole ring and the backbone amide groups of the third residue (His31 in 


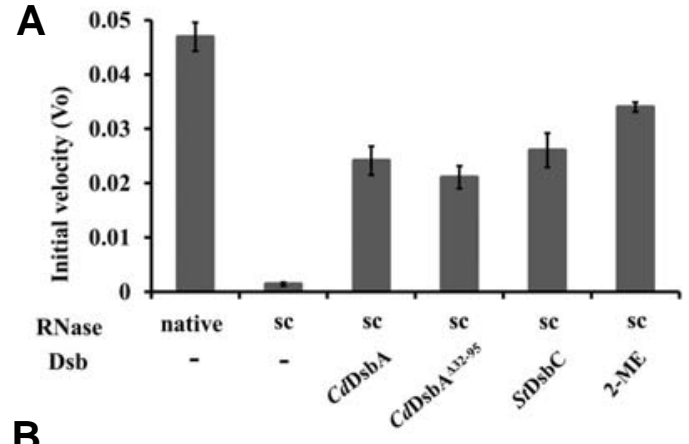

B

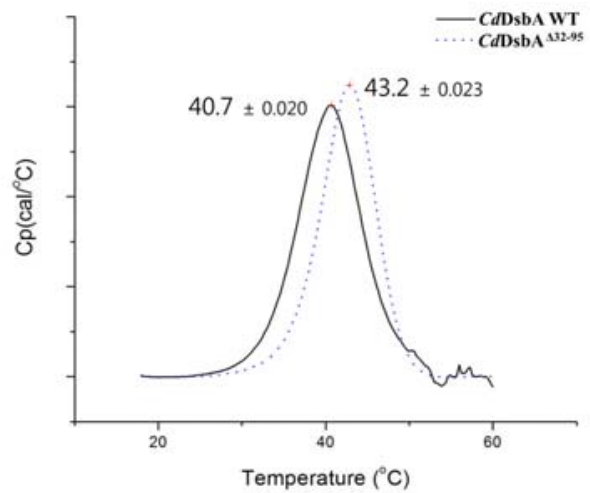

Fig. 4. Disulfide bond isomerase/reductase activity and thermostability of $\mathrm{CaDsbA}$ and its deletion variant. (A) Disulfide bond isomerase/reductase activity of CdDsbA was measured using denatured RNase A with scrambled disulfide bonds (sc or scRNase). The RNase A activity was measured at $\mathrm{pH} 7.5$ using the denatured

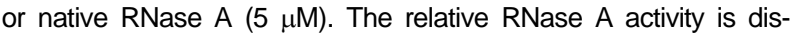
played relative to the activity from the same amount of the native RNase $A$ (native). Initial velocity $V_{0}$, for the folding of scRNase $A$ using CaDsbA (residues 32-289) and CaDsbA ${ }^{\Delta 32-95}$ (residues 96289) was compared with that of DsbC from $S$. enterica. serovar Typhimurium (StDsbC) and $2 \mathrm{mM}$ 2-mercaptoethanol (2-ME). All experiments were triplicated and the standard errors are indicated by error bars. (B) Overlay of DSC thermograms of the intact soluble domain of $\mathrm{CaDsbA}(\mathrm{CdDsbA} \mathrm{WT})$ and the $\mathrm{N}$-terminal regiondeleted $\mathrm{CaDsbA}\left(\mathrm{CdDsbA}{ }^{\triangle 32-95}\right)$. The structural stabilities of the proteins were evaluated by the $T_{m}$ values measured by DSC. The temperature ranged from 15 to $110^{\circ} \mathrm{C}$ and was scanned at a rate of $1^{\circ} \mathrm{C} / \mathrm{min}$. The thermograms above $60^{\circ} \mathrm{C}$ are omitted due to the noise from the irreversible denaturation of the proteins. The final thermograms were processed by subtracting the baseline from the sample thermograms. The red crosses display the peaks indicated by the analyzing program. The thermogram of $C a D s b A ~ W T$ is offset by $0.001 \mathrm{cal} /{ }^{\circ} \mathrm{C}$. The standard errors from three independent experiments are displayed as ' \pm value'.

$E c \mathrm{DsbA})$ in this motif form hydrogen bonds with $\mathrm{S} \gamma$ of the nucleophilic cysteine residue. In the CdDsbA structure, the similar interactions within the $\mathrm{CXXC}$ motifs were found except for the imidazole ring-mediated interaction because His31 in $E c D s b A$ is replaced with phenylalanine residue (Phe128) in CaDsbA (Fig. 3). Cys126 of CoDsbA makes five hydrogen bonds with adjacent backbone atoms (Fig. 3B). Additional polar interactions with the Thr248 in the Thr-cis-Pro motif were found in $\mathrm{CdDsbA}$. The sidechain hydroxyl group and backbone carbonyl group of Thr248 make hydrogen bonds with $\mathrm{S} \gamma$ of
Cys126 (Fig. 3A). From these interactions, we could speculate that these extensive polar interactions might be involved in stabilization of Cys126 thiolate form in CoDsbA like EcDsbA.

CdDsbA plays a role in the isomerization/reductive pathway similar to DsbC in Gram-negative bacteria

It was previously reported that $\mathrm{SaDsbA}$ functions as a disulfide bond isomerase/reductase like $\mathrm{DsbC}$ in Gram-negative bacteria (Heras et al., 2008). To investigate the activity of CdDsbA, we performed a disulfide bond isomerase/reductase assay using denatured RNase A with scrambled disulfide bonds as a substrate. CdDsbA mediated the reductive refolding of the denatured RNase A via its disulfide bond isomerase/reductase activity, which was validated with DsbC protein from $S$. enterica serovar Typhimurium and 2-mercaptoethanol employed as controls (Fig. 4A and Supplementary Fig. S1). Thus, our observation indicates that CoDsbA is involved in isomerization/reductive pathways like SaDsbA and DsbC of Gram-negative bacteria.

To investigate the role of the $\mathrm{N}$-terminal extended region, we produced a variant protein whose $\mathrm{N}$-terminal extended region was mostly deleted. The expression level of the mutant protein was very high, and the protein exhibited comparable activity to that of the intact soluble domain (residues 32-289) of $\mathrm{CdDsbA}$ (Fig. 4A and Supplementary Fig. S1). These results demonstrated that the $\mathrm{N}$-terminal extended region is not essential for the overall folding of the core DsbA-like domain and the isomerase/reductase activity of CdDsbA. We next carried out differential scanning calorimetry (DSC) to evaluate the role of the N-terminal region on the structural stability of the proteins. Specifically, wild-type $\mathrm{CdDsbA}$ and the $\mathrm{N}$-terminal region deleted variant proteins were applied to DSC. Figure 4B shows the $\mathrm{T}_{\mathrm{m}}$ values of the proteins (wild-type protein: $43.2^{\circ} \mathrm{C}$; deletion variant: $40.7^{\circ} \mathrm{C}$ ). The decreased $\mathrm{T}_{\mathrm{m}}$ of $2.5^{\circ} \mathrm{C}$ of the deletion variant versus the wild-type protein indicated that the $\mathrm{N}$-terminal extended region partly contributed to the structural stability of the core DsbA-like domain.

\section{CdCueP is a putative substrate of CdDsbA}

In the Gram-negative bacteria $S$. enterica serovar Typhimurium, the reduced form of CueP scavenges the production of hydroxyl radicals in the copper-mediated Fenton reaction, and DsbC is responsible for keeping CueP in the reduced, active form (Yoon et al., 2013; 2014a; 2014b). Given the disulfide bond isomerase/ reductase activity of $C d D s b A$, we investigated whether $C d D s b A$ shares a similar function with respect to reduction of CdCueP (accession number WP_003849917), which was discovered by the sequence search program BLAST (Altschul et al., 1990) (Supplementary Fig. S2). Specifically, we tested the activity of CoDsbA using NTB-labeled CdCueP as a substrate. The NTB group was attached to the cysteine residues of $\mathrm{CdCueP}$ using Ellman's reagent (the disulfide-containing agent 5,5'-dithiobis(2-nitrobenzoic acid) or DTNB), which can selectively react with the free cysteine (Yoon et al., 2014a). When freshly-prepared CoDsbA protein was incubated with NTB-labeled CdCueP, its reducing activity on CdCueP was as efficient as that of the strong reducing agent DTT (Fig. 5). In contrast, CaDsbA failed to reduce the NTB-labeled $S$. enterica CueP, which indicated that $C a D s b A$ recognized $C d C u e P$ via a specific protein-protein interaction. We next tested whether the reduced form of CdCueP can reduce CaDsbA using the NTB-labeled CaDsbA and the reduced form of CoCueP. Any significant reaction was not observed, indicating that $\mathrm{CdDsbA}$ is more reductive than $\mathrm{CdCueP}$ (Fig. 5). Thus, our findings suggest that DsbA is a putative reductant of CueP in the Gram-positive bacterium $C$. diphtheriae. 


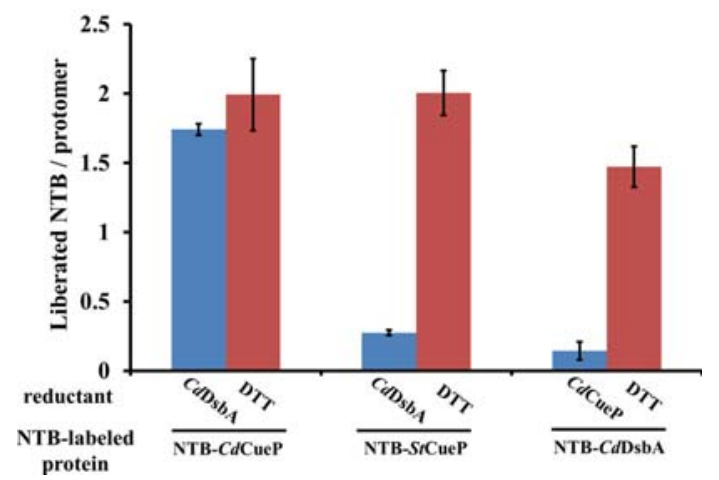

Fig. 5. The reducing activity of CaDsbA on CdCueP. NTB was attached to the CueP proteins from $C$. diphtheriae and $S$. enterica serovar Typhimurium or CaDsbA protein prior to the experiments. NTB-labelled proteins $(5 \mu \mathrm{M})$ were incubated with freshly-prepared CdDsbA or CdCueP $(15 \mu \mathrm{M})$ and DTT $(15 \mu \mathrm{M})$. The reducing activity was calculated based on the number of the liberated TNB from a CueP or DsbA protomer. All experiments were triplicated and the standard errors are indicated by error bars.

\section{DISCUSSION}

In this study, we identified genes encoding a DsbA homologous protein $(C d D s b A)$ and a CueP homologous protein (CdCueP) in the $C$. diphtheriae genome and determined the crystal structure of $\mathrm{CdDsbA}$ as well as the functional relationship between the two proteins. Despite structural differences, CaDsbA exhibited disulfide bond isomerase/reductase activity similar to that of DsbC from Gram-negative bacteria. Notably, a unique long Nterminal extended region was found in $\mathrm{CdDsbA}$, and the region was dispensable for the activity of the core DsbA-like domain. More importantly, this study showed that CaDsbA could reduce CueP of $C$. diphtheriae, but not of Salmonella, suggesting that CdCueP is a substrate of DsbA in C. diphtheria. However, further study is needed to know whether CdCueP is a physiological substrate of $C d D s b A$.

We noted that the long $\mathrm{N}$-terminal extended region of $\mathrm{CaDsbA}$ did not form notable hydrophobic interactions with the core DsbA-like domain. Without the $\mathrm{N}$-terminal region, the protein could be expressed in a soluble form with comparable enzymatic activity. Furthermore, the $\mathrm{N}$-terminal region only partly contributed to the structural stability of the protein. Thus, it is also expected that the $\mathrm{N}$-terminal extended region may be easily unfolded since it does not have an apparent secondary structure. This observation led us to ask what role the $\mathrm{N}$ terminal extended region might play. We speculated that the $\mathrm{N}$ terminal region may stretch to a long string (over $300 \AA$ ) from the anchored plasma membrane if the region is unfolded. Since DsbA is a chaperone protein that helps correct the folding of a diverse set of proteins, this long string from the anchoring membrane may provide a significant advantage in reaching substrates on the membrane.

How can CoDsbA maintain the reductase activity like DsbC of Gram-negative bacteria? To answer this question, we noted Etrx proteins from Streptococcus pneumoniae, which are extracellular surface thioredoxin-fold lipoprotein and functional orthologues of CdDsbaA. The Etrx proteins maintain methionine sulfoxide reductases with their reducing power, which plays an important role in extracellular ROS stress resistance of
S. pneumoniae (Saleh et al., 2013). Moreover, the Etrx proteins is in turn kept reduced by the membrane protein $\mathrm{CcdA}$, which has a low sequence similarity to $d s b D$ that supplies the reducing power to DsbC in Gram-negative bacteria (Saleh et al., 2013). The sequence analysis of $C$. diphtheriae revealed a putative $\mathrm{CcdA}$-like protein ( $31 \%$ sequence identity), suggesting that $\mathrm{CdDsbA}$ is kept in the reduced state by the CcdA-like proteins.

In S. enterica servar Typhimurium, DsbC and CueP are colocalized in the periplasm, and can make cooperative interactions in this confined space to scavenge the generation of hydroxyl radicals from $\mathrm{H}_{2} \mathrm{O}_{2}$ in the presence of copper ion (Yoon et al., 2014b). Since the Gram-positive bacterium C. diphtheriae does not have a conventional periplasmic space due to the lack of an outer membrane, many extracellular proteins are thought to be tethered at the extracellular side of the cytoplasmic membrane in order to function near the cell and make molecular interactions. Consistent with this possibility, the two $C$. diphtheriae proteins appeared to be tethered at the cytoplasmic membrane by a predicted uncleavable signal peptide or transmembrane helix. Given that both proteins seem to be anchored to the membrane, they are likely able to interact sufficiently to perform their functions. $C$ diphtheriae can survive in macrophage phagosomes, which is directly related to the pathogenesis of this bacterium. The role of DsbA in reduction of CueP in the extracellular region of $C$. diphtheriae might be involved in survival in macrophage phagosomes, since CueP is related to the survival of Salmonella in macrophage phagosomes (Yoon et al., 2014b).

In this study, the crystal structure of DsbA from C. diphtheriae was determined, which revealed a core DsbA-like domain and long extended $\mathrm{N}$-terminal region. The core domain of $\mathrm{CoDsbA}$ exhibited disulfide bond isomerase/reductase activity, while the $\mathrm{N}$-terminal extended region appeared to serve as a long anchor in the membrane. Furthermore, CdDsbA also showed reducing activity specifically toward CueP from $C$. diphtheriae.

Note: Supplementary information is available on the Molecules and Cells website (www.molcells.org).

\section{ACKNOWLEDGMENTS}

This study was supported by grants from the Korean Healthcare Technology R\&D Project, Korean Ministry of Health and Welfare (Grant no. H12C0947), and from the R\&D Convergence Center Support Program funded by the Ministry for Food, Agriculture, Forestry and Fisheries, Republic of Korea. This study made use of beamline $5 \mathrm{C}$ at the Pohang Accelerator Laboratory (Republic of Korea).

\section{REFERENCES}

Achard, M.E., Stafford, S.L., Bokil, N.J., Chartres, J., Bernhardt, P.V., Schembri, M.A., Sweet, M.J., and McEwan, A.G. (2012). Copper redistribution in murine macrophages in response to Salmonella infection. Biochem. J. 444, 51-57.

Adams, P.D., Grosse-Kunstleve, R.W., Hung, L.W., loerger, T.R., McCoy, A.J., Moriarty, N.W., Read, R.J., Sacchettini, J.C. Sauter, N.K., and Terwilliger, T.C. (2002). PHENIX: building new software for automated crystallographic structure determination. Acta Crystallogr. D. Biol. Crystallogr. 58, 1948-1954.

Altschul, S.F., Gish, W., Miller, W., Myers, E.W., and Lipman, D.J. (1990). Basic local alignment search tool. J. Mol. Biol. 215, 403410

Babior, B.M. (1999). NADPH oxidase: an update. Blood 93, 14641476.

Chen, Y., Yu, P., Luo, J., and Jiang, Y. (2003). Secreted protein prediction system combining CJ-SPHMM, TMHMM, and 
Crystal Structure and Function of DsbA

Si-Hyeon Um et al.

PSORT. Mamm. Genome 14, 859-865.

Cho, S.H., and Collet, J.F. (2013). Many roles of the bacterial envelope reducing pathways. Antioxid. Redox Signal. 18, 16901698.

Crow, A., Lewin, A., Hecht, O., Carlsson Moller, M., Moore, G.R., Hederstedt, L., and Le Brun, N.E. (2009). Crystal structure and biophysical properties of Bacillus subtilis BdbD. An oxidizing thiol:disulfide oxidoreductase containing a novel metal site. J. Biol. Chem. 284, 23719-23733.

Dorenbos, R., Stein, T., Kabel, J., Bruand, C., Bolhuis, A., Bron, S., Quax, W.J., and Van Dijl, J.M. (2002). Thiol-disulfide oxidoreductases are essential for the production of the lantibiotic sublancin 168. J. Biol. Chem. 277, 16682-16688.

Dumoulin, A., Grauschopf, U., Bischoff, M., Thony-Meyer, L., and Berger-Bachi, B. (2005). Staphylococcus aureus DsbA is a membrane-bound lipoprotein with thiol-disulfide oxidoreductase activity. Arch. Microbiol. 184, 117-128.

Erlendsson, L.S., and Hederstedt, L. (2002). Mutations in the thioldisulfide oxidoreductases $\mathrm{BdbC}$ and $\mathrm{BdbD}$ can suppress cytochrome $\mathrm{c}$ deficiency of CcdA-defective Bacillus subtilis cells. J. Bacteriol. 184, 1423-1429.

Goldstone, D., Haebel, P.W., Katzen, F., Bader, M.W., Bardwell, J.C., Beckwith, J., and Metcalf, P. (2001). DsbC activation by the $\mathrm{N}$-terminal domain of DsbD. Proc. Natl. Acad. Sci. USA 98, 9551-9556.

Guddat, L.W., Bardwell, J.C. and Martin, J.L. (1998). Crystal structures of reduced and oxidized DsbA: investigation of domain motion and thiolate stabilization. Structure 6, 757-767.

Haraga, A., Ohlson, M.B., and Miller, S.I. (2008). Salmonellae interplay with host cells. Nat. Rev. Microbiol. 6, 53-66.

Hatahet, F., Boyd, D., and Beckwith, J. (2014). Disulfide bond formation in prokaryotes: history, diversity and design. Biochim. Biophys. Acta 1844, 1402-1414

Heras, B., Kurz, M., Jarrott, R., Shouldice, S.R., Frei, P., Robin, G., Cemazar, M., Thony-Meyer, L., Glockshuber, R., and Martin, J.L. (2008). Staphylococcus aureus DsbA does not have a destabilizing disulfide. A new paradigm for bacterial oxidative folding. J. Biol. Chem. 283, 4261-4271.

Heras, B., Shouldice, S.R., Totsika, M., Scanlon, M.J., Schembri, M.A., and Martin, J.L. (2009). DSB proteins and bacterial pathogenicity. Nat. Rev. Microbiol. 7, 215-225.

Hiniker, A., Collet, J.F., and Bardwell, J.C. (2005). Copper stress causes an in vivo requirement for the Escherichia coli disulfide isomerase DsbC. J. Biol. Chem. 280, 33785-33791.

Hodgkinson, V., and Petris, M.J. (2012). Copper homeostasis at the host-pathogen interface. J. Biol. Chem. 287, 13549-13555.

Huber-Wunderlich, M., and Glockshuber, R. (1998). A single dipeptide sequence modulates the redox properties of a whole enzyme family. Fold Des. 3, 161-171

Jiao, L., Kim, J.S., Song, W.S., Yoon, B.Y., Lee, K., and Ha, N.C. (2013). Crystal structure of the periplasmic disulfide-bond isomerase DsbC from Salmonella enterica serovar Typhimurium and the mechanistic implications. J. Struct. Biol. 183, 1-10.

Kouwen, T.R., van der Goot, A., Dorenbos, R., Winter, T. Antelmann, H., Plaisier, M.C., Quax, W.J., van Dijl, J.M. and Dubois, J.Y. (2007). Thiol-disulphide oxidoreductase modules in the low-GC Gram-positive bacteria. Mol. Microbiol. 64, 984-999.

Martin, J.L. (1995). Thioredoxin--a fold for all reasons. Structure 3, 245-250.

Martin, J.L., Waksman, G., Bardwell, J.C., Beckwith, J. and Kuriyan, J. (1993). Crystallization of DsbA, an Escherichia coli protein required for disulphide bond formation in vivo. J. Mol. Biol. 230, 1097-1100.

Matias, V.R. and Beveridge, T.J. (2006). Native cell wall organization shown by cryo-electron microscopy confirms the existence of a periplasmic space in Staphylococcus aureus. J. Bacteriol. 188, 1011-1021.

Meima, R., Eschevins, C., Fillinger, S., Bolhuis, A., Hamoen, L.W., Dorenbos, R., Quax, W.J., van Dijl, J.M., Provvedi, R., Chen, I., et al. (2002). The bdbDC operon of Bacillus subtilis encodes thiol- disulfide oxidoreductases required for competence development. J. Biol. Chem. 277, 6994-7001.

Mohanty, P., Patel, A., and Kushwaha Bhardwaj, A. (2012). Role of $\mathrm{H}$ - and $\mathrm{D}$ - MATE-type transporters from multidrug resistant clinical isolates of Vibrio fluvialis in conferring fluoroquinolone resistance. PLoS One 7, e35752.

Nakamoto, H., and Bardwell, J.C. (2004). Catalysis of disulfide bond formation and isomerization in the Escherichia coli periplasm. Biochim. Biophys. Acta 1694, 111-119.

Osman, D., Patterson, C.J., Bailey, K., Fisher, K., Robinson, N.J., Rigby, S.E., and Cavet, J.S. (2013). The copper supply pathway to a Salmonella $\mathrm{Cu}, \mathrm{Zn}$-superoxide dismutase (SodClI) involves $\mathrm{P}(1 \mathrm{~B})$-type ATPase copper efflux and periplasmic CueP. Mol. Microbiol. 87, 466-477.

Otwinosky, Z., and Minor, W. (1997). Processing of X-ray diffraction data collected in oscillation mode. Methods Enzymol. 276, 307326.

Petersen, T.N., Brunak, S., von Heijne, G., and Nielsen, H. (2011). SignalP 4.0: discriminating signal peptides from transmembrane regions. Nat. Methods 8, 785-786.

Ren, G., Stephan, D., Xu, Z., Zheng, Y., Tang, D., Harrison, R.S. Kurz, M., Jarrott, R., Shouldice, S.R., Hiniker, A., et al. (2009). Properties of the thioredoxin fold superfamily are modulated by a single amino acid residue. J. Biol. Chem. 284, 10150-10159.

Rice, L.M., Earnest, T.N., and Brunger, A.T. (2000). Singlewavelength anomalous diffraction phasing revisited. Acta Crystallogr. D. Biol. Crystallogr. 56, 1413-1420.

Saleh, M., Bartual, S.G., Abdullah, M.R., Jensch, I., Asmat, T.M., Petruschka, L., Pribyl, T., Gellert, M., Lillig, C.H., Antelmann, H., et al. (2013). Molecular architecture of Streptococcus pneumoniae surface thioredoxin-fold lipoproteins crucial for extracellular oxidative stress resistance and maintenance of virulence. EMBO Mol. Med. 5, 1852-1870.

Sarvas, M., Harwood, C.R., Bron, S., and van Dijl, J.M. (2004). Post-translocational folding of secretory proteins in Grampositive bacteria. Biochim. Biophys. Acta 1694, 311-327.

Shouldice, S.R., Heras, B., Walden, P.M., Totsika, M., Schembri, M.A., and Martin, J.L. (2011). Structure and function of DsbA, a key bacterial oxidative folding catalyst. Antioxid. Redox Signal. $14,1729-1760$

Stein, T. (2005). Bacillus subtilis antibiotics: structures, syntheses and specific functions. Mol. Microbiol. 56, 845-857.

White, C., Lee, J., Kambe, T., Fritsche, K., and Petris, M.J. (2009). A role for the ATP7A copper-transporting ATPase in macrophage bactericidal activity. J. Biol. Chem. 284, 33949 33956.

Wolschendorf, F., Ackart, D., Shrestha, T.B., Hascall-Dove, L., Nolan, S., Lamichhane, G., Wang, Y., Bossmann, S.H., Basaraba, R.J., and Niederweis, M. (2011). Copper resistance is essential for virulence of Mycobacterium tuberculosis. Proc. Natl. Acad. Sci. USA 108, 1621-1626.

Yoon, B.Y., Kim, Y.H., Kim, N., Yun, B.Y., Kim, J.S., Lee, J.H., Cho, H.S., Lee, K., and Ha, N.C. (2013). Structure of the periplasmic copper-binding protein CueP from Salmonella enterica serovar Typhimurium. Acta Crystallogr. Sect. F Struct. Biol. Cryst. Commun. 69, 1867-1875.

Yoon, B.Y., Kim, J.S., Um, S.H., Jo, I., Yoo, J.W., Lee, K., Kim, Y.H., and Ha, N.C. (2014a). Periplasmic disulfide isomerase DsbC is involved in the reduction of copper binding protein CueP from Salmonella enterica serovar Typhimurium. Biochem. Biophys. Res. Commun. 446, 971-976.

Yoon, B.Y., Yeom, J.H., Kim, J.S., Um, S.H., Jo, I., Lee, K., Kim Y.H., and Ha, N.C. (2014b). Direct ROS Scavenging Activity of CueP from Salmonella enterica serovar Typhimurium. Mol. Cells 37, 100-108.

Yun, B.Y., Piao, S., Kim, Y.G., Moon, H.R., Choi, E.J., Kim, Y.O., Nam, B.H., Lee, S.J., and Ha, N.C. (2011). Crystallization and preliminary X-ray crystallographic analysis of Salmonella Typhimurium CueP. Acta Crystallogr. Sect. F Struct. Biol. Cryst. Commun. 67, 675-677. 